\title{
Atomic Positive Linear Maps in Matrix Algebras
}

By

\author{
Kil-Chan $\mathrm{HA}^{*}$
}

\begin{abstract}
We show that all of the known generalizations of the Choi maps are atomic maps.
\end{abstract}

\section{§1. Introduction}

Let $M_{n}$ be the $C^{*}$-algebra of all $n \times n$ matrices over the complex field and $\boldsymbol{P}_{s}\left[M_{n}\right]$ (respectively, $\boldsymbol{P}^{s}\left[M_{n}\right]$ ) the convex cone of all $s$-positive (respectively, $s$-copositive) linear maps between $M_{n}$. One of the basic problems about the structures of the positive cone $\boldsymbol{P}_{1}\left[M_{n}\right]$ is if this cone can be decomposed as the algebraic sum of some simpler subcones. It is well known $[22,25]$ that every positive linear map between $M_{2}$ is decomposable, that is, it can be written as the sum of a completely positive linear map and a completely copositive linear map. But, this is not the case for higher dimensional matrix algebras.

The first example of an indecomposable positive linear map in $M_{3}$ was given by Choi [5]. Choi and Lam [7] also gave an example of an indecomposable extremal positive linear map in $M_{3}$ (see also [6]). Another examples of indecomposable extremal positive linear maps are found in $[9$, 16, 21]. These maps are neither 2-positive nor 2-copositive, and so they become atomic maps in the sense in [24], that is, they can not be decomposed into the sums of 2-positive linear maps and 2-copositive linear maps. Several authors $[1,2,10,15,17,24]$ considered indecomposable positive linear maps as extensions of the Choi's example. The first two examples [2, 10] are generalizations of the Choi's map $[5,6]$ in $M_{3}$ and the other maps $\tau_{n, k}$ in $[1,15,17$, 24] (see Section 2 for the definition) are extensions of the Choi map [7] in

Communicated by T. Miwa, June 19, 1998.

1991 Math. Subject Classifications: 46L05, 15 A30.

partially supported by GARC-KOSEF

* Department of Mathematics, Seoul National University, Seoul 151-742, KOREA. 
higher dimensional matrix algebra $M_{n}$. Among them, examples in [10] and the map $\tau_{n, 1}(n \geq 3)$ [17] are known to be atomic maps. But the atomic properties of the other indecomposable maps are not determined. Even decomposabilities of the maps $\tau_{n, k}$ are remained open except for some special cases [17]. The usual method to show the atomic property of a positive linear map depends on a tedious matrix manipulation.

The purpose of this note is to show that all of the above mentioned examples are atomic maps, using the recent result of Eom and Kye [8]. Generalizing the Woronowicz's argument [25], they considered the duality between the space $M_{n} \otimes M_{m}\left(=M_{n m}\right)$ of all $n m \times n m$ matrices over the complex field and the space $\mathscr{L}\left(M_{m}, M_{n}\right)$ of all linear maps from $M_{m}$ into $M_{n}$, which is given by

$$
\langle A, \phi\rangle=\operatorname{Tr}\left[\sum_{i, j=1}^{m}\left(\phi\left(e_{i, j}\right) \otimes e_{i, j}\right) A^{\mathrm{t}}\right]=\sum_{i, j=1}^{m}\left\langle\phi\left(e_{i, j}\right), a_{i, j}\right\rangle,
$$

for $A=\sum_{i, j=1}^{m} a_{i, j} \otimes e_{i, j} \in M_{n} \otimes M_{m}$ and a linear map $\phi: M_{m} \rightarrow M_{n}$, where $\left\{e_{i, j}\right\}$ is the matrix units of $M_{m}$ and the bilinear form in the right-side is given by $\langle X, Y\rangle=\operatorname{Tr}\left(Y X^{t}\right)$ for $X, Y \in M_{n}$ with the usual trace $\operatorname{Tr}$. For the convenience of readers, we briefly explain the results in [8]. For a matrix $A=\Sigma_{i, j=1}^{m} x_{i, j} \otimes e_{i, j}$ $\in M_{n} \otimes M_{m}$, we denote by $A^{\mathrm{T}}$ the block-transpose $\sum_{i j=1}^{m} x_{j . i} \otimes e_{i, j}$ of $A$. We say that a vector $z=\sum_{i=1}^{m} z_{i} \otimes e_{i} \in C^{n} \otimes C^{m}$ is an s-simple if the linear span of $\left\{z_{1}, \cdots, z_{m}\right\}$ has the dimension $\leq s$, where $\left\{e_{1}, \cdots, e_{m}\right\}$ is the usual orthonormal basis of $C^{m}$. Let $V_{s}\left[M_{n}\right]$ (respectively $V^{s}\left[M_{n}\right]$ ) denote the convex cone in $M_{n} \otimes M_{n}$ generated by $z z^{*} \in M_{n} \otimes M_{n}$ (respectively $\left(z z^{*}\right)^{\mathrm{T}} \in M_{n} \otimes M_{n}$ ) with all $s$-simple vectors $z \in C^{n} \otimes C^{n}$. It turns out that $V_{s}\left[M_{n}\right]$ (respectively $V^{s}\left[M_{n}\right]$ ) is the dual cone of $\boldsymbol{P}_{s}\left[M_{n}\right]$ (respectively $\boldsymbol{P}^{s}\left[M_{n}\right]$ ) with respect to the pairing (1.1). With this machinery, the maximal faces of $\mathbb{P}_{s}\left[M_{n}\right]$ and $\mathbb{P}^{s}\left[M_{n}\right]$ are characterized in terms of $s$-simple vectors (see also $[12,13,14]$ ). Another consequence is a characterization of the cone $\boldsymbol{P}_{s}\left[M_{n}\right]+\boldsymbol{P}^{t}\left[M_{n}\right]$ : For a linear map $\phi: M_{n} \rightarrow M_{n}$, the map $\phi$ is the sum of an $s$-positive linear map and a $t$-copositive linear map if and only if $\langle A, \phi\rangle \geq 0$ for each $A \in V_{s}\left[M_{n}\right] \cap V^{t}\left[M_{n}\right]$. This result provides us a useful method to examine the atomic property for the generalizations of the Choi maps mentioned before.

Throughout this note, every vector in the space $C^{r}$ will be considered as an $r \times 1$ matrix. The usual orthonormal basis of $C^{r}$ and matrix units of $M_{r}$ will be denoted by $\left\{\mathrm{e}_{i}: i=1, \cdots, r\right\}$ and $\left\{e_{i, j}: i, j=1, \cdots, r\right\}$ respectively, regardless of the dimension $r$. 


\section{§2. The Maps $\tau_{n, k}$}

Let $\varepsilon$ be the canonical projection of $M_{n}$ to the diagonal part and $S$ be the rotation matrix in $M_{n}$ which sends $e_{i}$ to $e_{i+1}(\bmod n)$ for $i=1, \cdots, n$. The map $\tau_{n, k}: M_{n} \rightarrow M_{n}$ is defined by

$$
\tau_{n, k}(X)=(n-k) \varepsilon(X)+\sum_{i=1}^{k} \varepsilon\left(S^{i} X S^{* i}\right)-X, \quad X \in M_{n},
$$

for $k=1,2, \cdots, n-1$. The map $\tau_{n, 0}: M_{n} \rightarrow M_{n}$ is also defined by

$$
\tau_{n, 0}(X)=n \varepsilon(X)-X, \quad X \in M_{n} .
$$

It is easy to see that $\tau_{n, 0}$ is completely positive and $\tau_{n, n-1}$ is completely copositive. The positivity of $\tau_{n, k}$ is equivalent [1] to a certain cyclic inequality, which was shown by Yamagami [26]. The map $\tau_{3,1}$ is the Choi and Lam's example mentioned in the introduction. For $n \geq 4$, Osaka showed that $\tau_{n, n-2}$ is not the sum of a 3-positive linear map and a 3-copositive linear map [15], and $\tau_{n, 1}$ is atomic [17]. In this section, we show that the map $\tau_{n, k}$ is an atomic map for each $n \geq 3$ and $k=1,2, \cdots, n-2$.

For each fixed natural number $n=1,2, \cdots$, let $\left\{\omega_{i}: 1 \leq i \leq 3^{n}\right\}$ be the $3^{n}$-th roots of unity. Then we have

$$
\sum_{i=1}^{3^{n}} \omega_{i}^{k}=0, \quad 1 \leq k \leq 3^{n}-1
$$

For each $k=1,2, \cdots, n$, we define $m_{k} \in Z$ by $m_{k}=\frac{3}{2}\left(3^{k-1}-1\right)$. Then it is easy to see the following:

$$
m_{k}-m_{l}=m_{i}-m_{j} \text { if and only if }(k, i)=(l, j) \text { or }(k, l)=(i, j) .
$$

For any $\gamma>0$, we define $a_{i k}, c_{r} \in C^{n}$ by

$$
\begin{array}{ll}
a_{i 1}=\sum_{j=1}^{n} \omega_{i}^{m_{j}} e_{j}, & 1 \leq i \leq 3^{n}, \\
a_{i k}=\omega_{i}^{-m_{k}} a_{i 1}, & 1 \leq i \leq 3^{n}, \quad 2 \leq k \leq n, \\
c_{1}=e_{1}+\gamma e_{2}+\sum_{k=3}^{n-1} e_{k}+\frac{1}{\gamma} e_{n}, \\
c_{r}=S^{r-1} c_{1}, & 2 \leq r \leq n .
\end{array}
$$


For each $r=1,2, \cdots, n, i=1,2, \cdots, 3^{n}$ and $j=1,2, \cdots, n$, we define $b_{r i j} \in C^{n}$ by

$$
b_{r i j}= \begin{cases}a_{i j}, & j \neq r \\ c_{j} \circ a_{i j}, & j=r,\end{cases}
$$

where $\circ$ is the Schur product of $n \times 1$ matrices $c_{j}$ and $a_{i j}$. We also define $z_{r i} \in C^{n} \otimes C^{n}$ and $A_{r} \in M_{n} \otimes M_{n}$ by

$$
\begin{array}{ll}
z_{r i}=\sum_{j=1}^{n} b_{r i j} \otimes e_{j}, & 1 \leq r \leq n, 1 \leq i \leq 3^{n}, \\
A_{r}=\frac{1}{3^{n}} \sum_{i=1}^{3^{n}} z_{r i} z_{r i}^{*}, & 1 \leq r \leq n .
\end{array}
$$

Then we see that each $z_{r i}$ is a 2 -simple vector and so $A_{r} \in V_{2}\left[M_{n}\right]$. If we write $A_{r}$ by $A_{r}=\sum_{p, q=1}^{n}\left(A_{r}\right)_{p, q} \otimes e_{p, q} \in M_{n} \otimes M_{n}$, then it is easy to see that

$$
\left(A_{r}\right)_{p, q}= \begin{cases}e_{p, q}, & p \neq q, \\ \sum_{i=1}^{n} e_{i, i}, & p=q, p \neq r, \\ S^{r-1}\left(e_{1.1}+\gamma^{2} e_{2.2}+\sum_{i=3}^{n-1} e_{i, i}+\frac{1}{\gamma^{2}} e_{n, n}\right) S^{* r-1}, & p=q=r,\end{cases}
$$

by (2.1) and (2.2).

Now we define $A \in V_{2}\left[M_{n}\right]$ by $A=\frac{1}{n} \sum_{r=1}^{n} A_{r}=\sum_{p, q=1}^{n} A_{p, q} \otimes e_{p, q} \in M_{n} \otimes M_{n}$.

Then, by (2.3), we have

$$
A_{p, q}= \begin{cases}e_{p, q}, & p \neq q, \\ e_{1,1}+\frac{1}{n}\left(\gamma^{2}+(n-1)\right) e_{2,2}+\sum_{i=3}^{n-1} e_{i, i}+\frac{1}{n}\left(\frac{1}{\gamma^{2}}+(n-1)\right) e_{n, n}, & p=q=1, \\ S^{p-1} A_{1,1} S^{* p-1}, & p=q, p \neq 1 .\end{cases}
$$

In order to show that $A \in V^{2}\left[M_{n}\right]$, define $u_{i}, v_{i}, \alpha_{i}$ and $\beta_{i j} \in C^{n}$ by

$$
\begin{array}{ll}
u_{i}=\frac{\gamma}{\sqrt{n}} e_{i+1} \otimes e_{i}+\frac{1}{\sqrt{n \gamma}} e_{i} \otimes e_{i+1}, & 1 \leq i \leq n, \\
v_{i}=\sqrt{\frac{n-1}{n}} e_{i+1} \otimes e_{i}+\sqrt{\frac{n-1}{n}} e_{i} \otimes e_{i+1}, & 1 \leq i \leq n,
\end{array}
$$




$$
\begin{array}{ll}
\alpha_{i}=e_{i} \otimes e_{i} & 1 \leq i \leq n, \\
\beta_{1 j}=e_{j} \otimes e_{1}+e_{1} \otimes e_{j}, & 3 \leq j \leq n-1, \\
\beta_{i j}=e_{j} \otimes e_{i}+e_{i} \otimes e_{j}, & 2 \leq i \leq(n-2),(i+2) \leq j \leq n,
\end{array}
$$

where suffixes are understood in $\bmod n . \quad$ A direct calculation shows

$$
A^{\mathrm{T}}=\sum_{i=1}^{n}\left(u_{i} u_{i}^{*}+v_{i} v_{i}^{*}+\alpha_{i} \alpha_{i}^{*}\right)+\sum_{j=3}^{n-1} \beta_{1 j} \beta_{1 j}^{*}+\sum_{i=2}^{n-2} \sum_{j=i+2}^{n} \beta_{i j} \beta_{i j}^{*}
$$

and so, we have $A \in V^{2}\left[M_{n}\right]$. Furthermore, we also have $\left\langle A, \tau_{n, k}\right\rangle=\gamma^{2}-1$, for each $n=3,4, \cdots$ and $k=1,2, \cdots, n-2$, and so we see that $\left\langle A, \tau_{n, k}\right\rangle<0$ for $0<\gamma<1$. By the result in [8] mentioned in the introduction, we conclude the following:

Theorem 2.1. For $n \geq 3$ and $1 \leq k \leq n-2$, the map $\tau_{n, k}: M_{n} \rightarrow M_{n}$ is an atomic positive linear map.

\section{§. The Generalized Choi Map}

The other generalization of the Choi map is given in [2]. For nonnegative real numbers $a, b$, and $c$, the map $\Phi[a, b, c]: M_{3} \rightarrow M_{3}$ is defined by

$\Phi[a, b, c](X)=$

$$
\left(\begin{array}{ccc}
a x_{1,1}+b x_{2,2}+c x_{3.3} & 0 & 0 \\
0 & a x_{2,2}+b x_{3,3}+c x_{1,1} & 0 \\
0 & 0 & a x_{3,3}+b x_{1,1}+c x_{2,2}
\end{array}\right)-X
$$

for each $X=\left(x_{i, j}\right) \in M_{3}$. The map $\Phi[2,0, \mu]$ with $\mu \geq 1$ is the example of an indecomposable positive linear map given by Choi [6], and $\Phi[2,0,2]$ and $\Phi[2,0,1]$ are the examples given in [5] and [7] respectively. Choi and Lam [7] showed that $\Phi[2,0,1]$ is an extremal positive linear map using the theory of biquadratic forms. Later, Tanahashi and Tomiyama [24] showed the atomic property of the map $\Phi[2,0,1]$ which is same as the map $\tau_{3,1}$. It was shown [2] that the map $\Phi[a, b, c]$ is an indecomposable positive linear map if and only if the following conditions are satisfied: 

(i) $1 \leq a<3$,
(ii) $a+b+c \geq 3$,
(iii) $\begin{cases}(2-a)^{2} \leq b c<\left(\frac{3-a}{2}\right)^{2} & \text { if } 1 \leq a \leq 2 \\ 0 \leq b c<\left(\frac{3-a}{2}\right)^{2} & \text { if } 2 \leq a<3 .\end{cases}$

In this section we show that these conditions imply that $\Phi[a, b, c]$ is an atom.

Let $\left\{\omega_{i}: i=1,2,3\right\}$ be the cube roots of unity and $s$ be any positive real number. Define $a_{i k} \in C^{3}, z_{i}, u_{i} \in C^{3} \otimes C^{3}$ and $B \in V_{2}\left[M_{3}\right]$ by

$$
\begin{gathered}
a_{i 1}=\left(\omega_{i}, 0,0\right)^{\mathrm{t}}, \quad a_{i 2}=\left(0, \omega_{i}, \frac{\omega_{i}^{2}}{s}\right)^{\mathrm{t}}, \quad a_{i 3}=\left(\frac{s}{\omega_{i}}\right) a_{i 2}, \quad i=1,2,3, \\
z_{i}=\sum_{k=1}^{3} a_{i k} \otimes e_{k}, \quad i=1,2,3 \\
u_{1}=e_{2} \otimes e_{1}, \quad u_{2}=e_{1} \otimes e_{3}, \quad u_{3}=e_{3} \otimes e_{1}, \quad u_{4}=e_{1} \otimes e_{2}, \\
B=\frac{1}{3}\left(\sum_{i=1}^{3} z_{i} z_{i}^{*}\right)+\frac{1}{s^{2}}\left(\sum_{i=1}^{2} u_{i} u_{i}^{*}\right)+s^{2}\left(\sum_{i=3}^{4} u_{i} u_{i}^{*}\right) .
\end{gathered}
$$

It is clear that $B \in V_{2}\left[M_{3}\right]$. To show that $B \in V^{2}\left[M_{3}\right]$, we define $z_{i}$ and $u_{i} \in C^{3} \otimes C^{3}$ by

$$
\begin{array}{ll}
z_{i}=\frac{1}{s}\left(e_{i+1} \otimes e_{i}\right)+s\left(e_{i} \otimes e_{i+1}\right), & i=1,2,3, \\
u_{i}=e_{i} \otimes e_{i}, & i=1,2,3,
\end{array}
$$

where suffixes are understood in mod 3. A direct calculation show that

$$
B^{\mathrm{T}}=\sum_{i=1}^{3}\left(z_{i} z_{i}^{*}+u_{i} u_{i}^{*}\right) \in V_{2}\left[M_{3}\right]
$$

It is also easy to calculate

$$
\langle B, \Phi[a, b, c]\rangle=3\left((a-3)+\frac{c}{s^{2}}+s^{2} b\right) .
$$


We proceed to show the conditions in (3.1) imply that the pairing in (3.2) is negative. We first consider the case $b c=0$. If $b=0$ then the pairing (3.2) becomes negative for $s>\sqrt{\frac{c}{3-a}}$. If $c=0$ then (3.2) is negative for $0<s<\sqrt{\frac{3-a}{b}}$. When $b c \neq 0$, we take $s=\left(\frac{c}{b}\right)^{1 / 4}$, then the pairing (3.2) is reduced to

$$
\langle B, \Phi[a, b, c]\rangle=3((a-3)+2 \sqrt{b c}),
$$

which is also negative since $\sqrt{b c}<\frac{3-a}{2}$ in (3.1). Therefore we have Theorem 3.1

Theorem 3.1. The map $\Phi[a, b, c]$ is an indecomposable positive linear map if and only if it is an atomic positive linear map.

For the Choi's map $\Phi[2,0, \mu]$, the condition (3.1) is reduced to the condition $\mu \geq 1$. Therefore, we see that the Choi's map $\Phi[2,0, \mu]$ is atomic whenever $\mu \geq 1$.

\section{§4. The Robertson's Map}

An example of an indecomposable positive linear map on $M_{4}$ was given by Robertson [18] by considering an extension of an automorphism on a certain spin factors. To describe this map, let $\sigma: M_{2} \rightarrow M_{2}$ be the symplectic involution defined by

$$
\sigma\left(\begin{array}{ll}
\alpha & \beta \\
\gamma & \delta
\end{array}\right)=\left(\begin{array}{cc}
\delta & -\beta \\
-\gamma & \alpha
\end{array}\right)
$$

The Robertson's map $\Psi: M_{4} \rightarrow M_{4}$ is defined by

$$
\Psi\left(\begin{array}{cc}
X & Y \\
Z & W
\end{array}\right)=\left(\begin{array}{cc}
\operatorname{tr}(W) I_{2} & \frac{1}{2}(Y+\sigma(Z)) \\
\frac{1}{2}(Z+\sigma(Y)) & \operatorname{tr}(X) I_{2}
\end{array}\right)
$$

for $X, Y, Z, W \in M_{2}$, where $\operatorname{tr}$ is the normalized trace on $M_{2}$. The indecomposability of this map was shown in [20] by using the Størmer's characterization [23] of decomposability.

It turns out that this map is an extremal positive linear map which is neither 2-positive nor 2-copositive [19,21]. So this map is an atomic map. We 
provide a simple proof.

Define $z_{i} \in \mathbb{C}^{4} \otimes \mathbb{C}^{4}$ and $D \in \mathbb{V}_{2}\left[M_{4}\right]$ by

$$
\begin{gathered}
z_{1}=e_{1} \otimes e_{1}, \quad z_{2}=e_{1} \otimes e_{3}, \quad z_{3}=e_{2} \otimes e_{1}, \quad z_{4}=e_{2} \otimes e_{4}, \\
z_{5}=e_{3} \otimes e_{1}, \quad z_{6}=e_{3} \otimes e_{3}, \quad z_{7}=e_{3} \otimes e_{4}, \\
D=\left(z_{1}-z_{6}\right)\left(z_{1}-z_{6}\right)^{*}+\left(z_{5}+z_{4}\right)\left(z_{5}+z_{4}\right)^{*}+z_{2} z_{2}^{*}+z_{3} z_{3}^{*}+z_{7} z_{7}^{*} .
\end{gathered}
$$

Then we see that

$$
D^{\mathrm{T}}=\left(z_{5}-z_{2}\right)\left(z_{5}-z_{2}\right)^{*}+\left(z_{3}+z_{7}\right)\left(z_{3}+z_{7}\right)^{*}+z_{1} z_{1}^{*}+z_{6} z_{6}^{*}+z_{4} z_{4}^{*} \in \mathbb{V}_{2}\left[M_{4}\right],
$$

and so $D \in \mathbb{V}_{2}\left[M_{4}\right] \cap \mathbb{V}^{2}\left[M_{4}\right]$. Furthermore, we can show that the pairing $\langle D, \Psi\rangle=-\frac{1}{2}$ by an easy calculation. Consequently, we conclude that $\Psi$ is an atomic map.

\section{References}

[1] Ando, T., Positivity of certain maps, Seminar Notes, 1985.

[2] Cho, S. J., Kye, S.-H. and Lee, S. G., Generalized Choi maps in 3-dimensional matrix algebras, Linear Alg. Appl., 171 (1992), 213-224.

[3] Choi, M.-D., Positive linear maps on $C^{*}$-algebras, Canad. Math. J., 24 (1972), 520-529.

[4] Completely positive linear maps on complex matrices, Linear Alg. Appl., 10 (1975), 285-290.

[5] - Positive semidefinite biquadratic forms, Linear Alg. Appl., 12 (1975), 95-100.

[6] Some assorted inequalities for positive linear maps on $C^{*}$-algebras, J. Operator Theory, 4 (1980), 271-285.

[7] Choi, M.-D. and Lam, T. Y., Extremal positive semidefinite forms, Math. Ann., 231 (1977), 1-18.

[8] Eom, M. H. and Kye, S.-H., Duality for positive linear maps in matrix algebras, Math. Scand., to appear.

[9] Kim, H.-J. and Kye, S.-H., Indecomposable extreme positive linear maps in matrix algebras, Bull. London Math. Soc., 26 (1994), 575-581.

[10] Kye, S.-H., Elementary Operators and Applications, World-Scientific, 1992.

[11] , Positive linear maps between Matrix algebras which fix diagonals, Linear Alg. Appl., 216 (1995), 239-256.

[12] , Facial structures for positive linear maps between matrix algebra, Canad. Math. Bull., 39 (1996), 74-82.

[13] , Boundaries of the cone of positive linear maps and its subcones in matrix algebras, J. Korean Math. Soc., 33 (1996), 669-677.

[14] On the convex set of all completely positive linear maps in matrix algebras, Math. Proc. Camb. Phil. Soc., 122 (1997), 45-54.

[15] Osaka, H., Indecomposable positive maps in low dimensional matrix algebras, Linear Alg. Appl., 153 (1991), 73-83.

[16] - A class of extremal positive maps in $3 \times 3$ matrix algebras, Publ. RIMS. Kyoto Univ., 28 (1992), 747-756. 
[17] Osaka, H., A series of absolutely positive maps in matrix algebras, Linear Alg. Appl., 186 (1993), 45-53.

[18] Robertson, A. G., Automorphisms of spin factors and the decomposition of positive maps, Quart. J. Math. Oxford (2), 34 (1983), 87-96.

[19] - Positive extensions of automorphisms of spin factors, Proc. Roy. Soc. Edinburgh Sect. $A, 94$ (1983), 71-77.

[20] Schwarz inequalities and the decomposition of positive maps on $C^{*}$-algebras, Math. Proc. Camb. Phil. Soc., 94 (1983).

[21] - Positive projections on $C^{*}$-algebra and an extremal positive map, J. London Math. Soc., 32 (1985), 133-140.

[22] Størmer, E., Positive linear maps of operator algebras, Acta Math., 110 (1963), 233-278.

[23] - Decomposable positive maps on $C^{*}$-algebras, Proc. Amer. Math. Soc., 86 (1982), 402-404.

[24] Tanahashi, K. and Tomiyama, J., Indecomposable positive maps in matrix algebras, Canad. Math. Bull., 31 (1988), 308-317.

[25] Woronowicz, S. L., Positive maps of low dimensional matrix algebras, Rep. Math. Phys., 10 (1976), 165-183.

[26] Yamagami, S., Cyclic inequalities, Proc. Amer. Math. Soc., 118 (1993), 521-527. 
